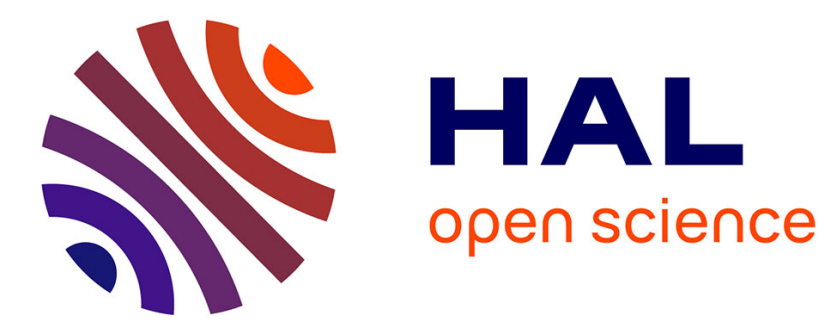

\title{
L'INSOLUBILISATION PROGRESSIVE DES POUDRES DE LAIT
}

\author{
M. Marc Fouassier
}

\section{To cite this version:}

M. Marc Fouassier. L'INSOLUBILISATION PROGRESSIVE DES POUDRES DE LAIT. Le Lait, 1924, 4 (35), pp.366-369. hal-00894767

\section{HAL Id: hal-00894767 https://hal.science/hal-00894767}

Submitted on 1 Jan 1924

HAL is a multi-disciplinary open access archive for the deposit and dissemination of scientific research documents, whether they are published or not. The documents may come from teaching and research institutions in France or abroad, or from public or private research centers.
L'archive ouverte pluridisciplinaire HAL, est destinée au dépôt et à la diffusion de documents scientifiques de niveau recherche, publiés ou non, émanant des établissements d'enseignement et de recherche français ou étrangers, des laboratoires publics ou privés. 
que c'est du phosphate de calcium et sans doute aussi de magnésium insoluble qui forme le constituant principal de la matière minérale précipitée.

\title{
L'INSOLUBILISATION PROGRESSIVE DES POUDRES DE LAIT,
}

\author{
par M. MARc FOUASSIER,
} de l'Institut Pasteur.

L'insolubilisation progressive qu'accusent certaines poudres de lait, peu de temps après. leur fabrication, est un phénomène bien connu que déplorent tous ceux qui s'intéressent à ce produit, car cette grave modification dans l'état de la poudre se répercute sur son utilisation.

En effet, ces poudres insolubles ou partiellement solubles ve sauraient être employées pour l'alimentation directe des adultes, à plus forte raison des enfants, soit seules, soit incorporées à des féculents ou à du cacao, leur emploi à cet état étant subordonné à leur solubilité ; leur emploi en biscuiterie ou en boulangerie n'offre pas par contre d'inconvénients sérieux,

L'insolubilisation m'est apparue particulièrement rapide pour les poudres obtenues par le procédé de dessiccation du lait répandu en nappes sur des cylindres chauffés. La pellicule lactée est, on le sait, détachée du cylindre à chaque révolution de celui-ci et ensuite tamisée, puis ensachée. Elle m'est apparue au contraitre très lente,et même insensible, après une année, pour une poudre obtenue par le procédé de pulvérisation du lait en chambre chaude, comme e'est le cas pour certaines poudres américaines conservées après fabrication dans des boîtes en fer blanc.

Il n'est pas dans mon intention de démontrer dans cet exposé la supériorité d'une poudre de lait quelconque ou d'un procédé de fabrieation, la comparaison entre une poudre obtenue par le procédé des cylindres et une poudre obtenue par pulvérisation ne devant servir qu'à donner l'explication d'un phénomène biologique qui a particulièrement retenu mon attention et qui est susceptible d'intéresser de nombreux fabricants. C'est à ce titre que je crois utile d'exposer plus largement ici les résultats d'un travail publié par ailleurs (1).

Selon le procédé de fabrication employé, la poudre de lait a un aspect très différent. Examinée au microscope, elle se présente en

(1) Bulletin Soc. Chimie Biol., juin 1923. 
effet sous forme de masses amorphes de différentes grosseurs aux contours déchiquetés : c'est le cas pour la poudre obtenue par le procédé des cylindres, que je désignerai sous le nom de poudre A, par opposition à la poudre obtenue par pulvérisation que je désignerai sous le nom de poudre $B$. Cette dernière examinée dans les mêmes conditions, se présente sous l'aspect de petits grains ronds qui ont conservé la forme vésiculaire des gouttelettes originelles.

La poudre A est grossière et rêche au toucher, tandis que la poudre $B$ est extrêmement finè et fuit sous le doigt par suite de sa texture.

Les facteurs susceptibles, à première vue, d'avoir une influence sur la solubilité de la poudre de lait, me semblent devoir être tout d'abord envisagés ; ce sont : la température de dessiceation et l'acidité du lait.

La température de dessiccation dans le procédé des cylindres atteint $120^{\circ}$; en réalité, cette température est celle à laquelle sont portés les cylindres, ce n'est pas celle subie par le lait qui atteint au plus $100^{\circ}$. Les albumines sont certes altérées par cette température, mais la caséine ne saurait être gravement influencée.

Quant à l'acidité du lait, j'ai pu me rendre compte qu'une poudre provenant d'un lait frais, d'acidité normale, perdait peu à peu sa solubilité, celle-ci devenait même nulle au bout de deux mois.

Des essais préliminaires de conservation de la poudre $\mathrm{A}$, fraîchement préparée, puis soumises en vases clos ou ouverts à diverses influences de températures ou d'humidité, ne m'ont pas donné de résultats satisfaisants en aucun eas, je n'ai pu empêcher le phénomène d'insolubilisation de se manifester, il m'a été donné au contraire de l'accélérer en certaines circonstances.

Instruit par ces résultats, j'ai établi une série d'expériences sur la poudre B qui avait conservé toute sa solubilité longtemps après fabrication afin de rechercher les conditions requises pour la rendre insoluble.

Expérience I. - Dans trois petits vases en verre fermant à l'émeri, dénommés pèses-filtres, j'ai introduit une même quantité de poudre B (2 gr.). La cavité intérieure du couverele de chaque récipient était garnie d'une épaisseur circulaire de papier filtre, n'ayant à aucun moment çontact avee la poudre. Le premier papier a été imprégné de einq gouttes d'eau, le second de cinq gouttes d'ammoniaque et le troisième ne reçut aucune imprégnation. Les trois récipients, clos hermétiquement, furent ensuite portés avec leur contenu à l'étuve à $37^{\circ}$. 
Les poudres furent examinées au bout de 12 heures. J'ai remarqué alors que seule la première avait perdu une grande partie de sa solubilité. Au bout de 24 heures, la poudre en présence de l'humidité était complètement insoluble, tandis que les deus autres étaient. encore intégralemant solubles.

Expérience II. - Une même quantité de poudre B $(1 \mathrm{gr}$.$) a été$ introduite dans une série de tubes à essais, ceux-ci reçurent ensuite une quantité d'eau progressive, de 1 à 10 gouttes. Ces tubes, en présence de témoins non humidifiés, ont été plongés pendant cinq minutes dans de l'eau bouillante. Après ce temps, chaque túbe a reçu $10 \mathrm{~cm}^{3}$ d'eau pour pouvoir constater le degré de solubilité. J'ai pu ainsi observer qu'une seule goutte d'eau était suffisante pour diminuer la solubilité de la poudre par rapport au témoin, que cette insolubilité progressait en fonction de l'humidité et qu'elle devenait totale pour le tube qui avait reçu cinq gouttes d'eau, tandis qu'à partir de la dose de 9 gouttes d'eau, elle commençait à se manifester faibiement à nouveau.

Le coefficient d'insolubilité passe donc par un maximum, mais $5 \%$ environ d'humidité suffisent pour que la poudre commence à deveriir insoluble.

Ces résultats, confirmés pour une poudre A de récente fabrication, permettent d'expliquer le mécanisme de l'insolubilité progressive des poudres de tait. Celle-ci ne serait en effet qu'une conséquence de la précipitation de la caséine par l'acide lactique, exactement du même ordre que celle qui se produit dans un lait arrivé à un certain degré d'acidité sous linfluence microbienne. Si, dans le cas que j'envisage, l'intervention microbienne ne joue aucun rỏle, ainsi que le prouve l'expérience II, il ne faut pas oublier cependant que la fonction acide naturelle du lait frais, correspondant à environ $1 \mathrm{gr} ., 8$ d'acide lactique par litre, cst conservée intégralement dans la poudre de lait et se trouve dans un même rapport avec l'extrait sec. Cette quantité d'acide, diluée dans le lait, est, du fait même de cette dilution 1,8/1033, sans action sur la caséine; elle est également sans action en milieu alcalin sur une poudre légèrement humide; elle est encore sans action lorsqu'elle se trouve à l'état anbydre en présence d'une caséine sèche dans le rapport 1,8/100 (qui correspond à celui d'une poudre de lait écrémé); mais. en présence d'humidité qui favorise son contact plus intime avec la caséine, la fonction acide se manifeste. Cette action sera d'autant plus profonde que l'acide se trouvera à un état de concentration favorable ; celle-ci diminuant, l'acidité perd son activité, ce qui explique la réapparition de solubilité partielle de la poudre à un certain degré d'humidification.

La manifestation de l'acidité naturelle du lait a été accélérée dans 
mes expériences par la chaleur, elle se manifeste de mème mais plus lentement au contact de l'air ambiant en fonction de la température et de l'humidité : c'est le cas de la pratique.

Il me paraît intéressant d'illustrer cette explication par un exemple frappant. Supposons en effet que l'on mélange intimement dans un flacon sec, du bicarbonate de soude et de l'acide tartrique, l'un et l'autre finement pulvérisés et très secs. Malgré la vive affinité des produits en contact, aucun phénomène ne se produira tant qu'on les conservera à l'abri de l'humidité. Faisons tomber une goutte d'eau en un point quelconque du mélange: immédiatenent l'acide tartrique agira activement sur le bicarbonate à ce seul point. Ouvrons maintenant le flacon et laissons-le débouché, exposé à l'air. L'humidité de l'air ambiant se fixera sur nos produits et favorisera leur contact, progressivement, et beaucoup plus lentement que dans le cas de la goutte d'eau, l'acide tartrique réagira sur le bicarbonate qui sera à la longue totalement décomposé.

Le taux d'humidité fixée ou non éliminée, joue done un rôle très important sur la persistance de la solubilité des poudres de lait. La poudre A que j'ai utilisée contenait $6.8 \%$ d'eau, condition favorable pour provoqner peu à peu son insolubilité, comme je l'ai constaté, tandis que la poudre $\mathrm{B}$ contenait seulement $1,6 \%$ d'humidité, ce qui explique sa longue conservation. Comme dans l'exemple ci-dessus, et ainsi que je l'ai démontré dans mes expériences, l'humidité acquise ou non suffisamment éliminée au moment de la fabrication, favorise, ou, mieux encore, provoque l'action insolubilisante de l'acide lactique sur la caséine de la poudre de laịt.

Il est aisé de tirer une conclusion pratique de cet exposé. Le fabricant de poudre de lait, qui poursuivrale but d'obtenir une poudre susceptible de conserver toute sa solubilité,devra, lors de la fabrication,réduire le taux d'humidité à environ $3 \%$ et maintenir ce taux constant dans le produit livré au commerce, par un emballage soigné, afin d'éviter toute humidification au contact de l'air.

\title{
LE PROGEDE IRRAUSE POUR LA FABRICATION DE LA POUDRE DE LAIT,
}

\author{
par LÉon PANCHAUD,
}

Docteur ès Sciences, attaché au Laboratoire Cantonal d'Analyses de Genève (Suisse).

Depuis quelques années (1918), les périodiques de langue allemande consacrent de nombreux articles aux différents produits (poudre de lait, poudre de sang, poudre d'œufs, etc...) obtenus par 